\title{
EOPs: Estrategias farmacológicas para la discontinuación del uso de benzodiacepinas
}

\author{
Pharmacologic strategies for benzodiazepine discontinuation
}

Fanny Rodríguez Santos *

\begin{abstract}
Resumen
En el presente artículo se evalúa la evidencia existente acerca de la eficacia de intervenciones farmacológicas y no farmacológicas para la discontinuación de benzodiacepinas en pacientes que las consumen de forma crónica. Se realiza una pequeña introducción al tema, se describe la estrategia de búsqueda, se resume una revisión sistemática que intentó responder la pregunta planteada y se expresan las conclusiones.

La información disponible sugiere que la reducción gradual es preferible a la interrupción abrupta, sugiriendo el uso eventual de carbamazepina como coadyuvante.

\section{Abstract}

In this article we evaluate the existing evidence about the effectiveness of pharmacological and nonpharmacological interventions for the discontinuation of benzodiazepines in patients who chronically consume them. It takes a brief introduction about the topic, describes the search strategy, summarizes a systematic review attempted to answer the question and the conclusions are expressed.

The available information suggests that gradual reduction is preferable to abrupt discontinuation, suggesting possible use of carbamazepine as an adjunct therapy.
\end{abstract}

Palabras clave: benzodiacepinas, discontinuación, adicción. Key words: benzodiazepines, discontinuation, adiction.

Rodríguez Santos F. Estrategias farmacológicas para la discontinuación del uso de benzodiacepinas. Evid. Act. Pract. Ambul. 12(2). 73-74. Abr-Jun. 2009.

\section{Escenario clínico}

Un médico de familia atiende a una mujer de 64 años. Durante la consulta la paciente refiere que toma lorazepam $2 \mathrm{mg}$ por día hace un año y medio. Otro profesional se la había prescripto porque, tras la muerte de su marido, padecía insomnio. Había intentado suspender la medicación ocho meses atrás, pero volvió a consumirla porque no podía dormir.

El médico destaca la importancia de que esta paciente abandone definitivamente las benzodiazepinas y se pregunta qué estrategias farmacológicas pueden contribuir a que pueda lograrlo.

\section{Pregunta que generó el caso}

En pacientes adultos que reciben tratamiento crónico con benzodiazepinas (población) ¿qué intervenciones farmacológicas y no farmacológicas resultan más eficaces (intervención) en el tratamiento de la dependencia/ discontinuación a estas drogas (resultado)?

\section{Introducción}

Las Benzodiazepinas pertenecen al grupo de medicamentos llamados depresores del sistema nervioso central (SNC). Son agentes sedantes-hipnóticos y fueron introducidos por primera vez en 1960. A partir de entonces y gracias a su mejor perfil de seguridad en comparación con los barbitúricos -que presentan más interacciones con otros fármacos y efectos adversos- su tasa de prescripción ha ido en ascenso hasta la actualidad.

Si bien la prevalencia de su consumo en la población general presenta grandes variaciones entre los países, en general se mantiene en tasas elevadas. Entre el 10 y el $20 \%$ de la población de los países occidentales reconoce haber consumido benzodiazepinas durante el último año, y entre el 1 y el $3 \%$ las ha consumido diariamente durante más de un año. En general se observa un mayor consumo en mujeres y en personas de 50 a 65 años, siendo principalmente indicados para el tratamiento de los trastornos de ansiedad y del insomnio. Sin embargo, se ha demostrado que son fármacos potencialmente adictivos y pueden proporcionar un reforzamiento en algunos individuos. En este sentido, se estima que aproximadamente la mitad de las personas que luego de un año con- tinúan tomando benzodiazepinas, lo hacen debido a la dependencia física y psicológica que este consumo ha producido y no debido a los efectos médicos y terapéuticos de la droga.

\section{Estrategia de búsqueda}

Se realizó una búsqueda avanzada en PubMed limitándola a revisiones sistemáticas o metanálisis con las palabras "benzodiazepine" and "dependence" and "discontinuation". La búsqueda arrojó 42 citas, cinco de las cuales resultaron pertinentes para responder la pregunta. Sin embargo, de éstas sólo una ofreció facilidades para acceder al artículo:

- Denis C y col. Pharmacological interventions for benzodiazepine mono-dependence management in outpatient settings. Cochrane Database of Systematic Reviews 2006, Issue 3. Art. No.: CD005194. DOI: 10.1002/14651858.CD005194.pub2.

Se repitió la estrategia de búsqueda en TripDataBase y se rea-lizó una búsqueda en Cochrane Database of Systematic Reviews con las palabras "benzodiazepine" and "dependence". En ambas la única cita pertinente fue la revisión antes nombrada.

\section{Resumen de la evidencia}

Los autores de este trabajo cuya última modificación significativa data de 2006 realizaron búsquedas en el Registro Especializado de Ensayos Controlados del Grupo Cochrane de Drogas y Alcohol (2004) en el Registro Cochrane Central de Ensayos Controlados, en MEDLINE (1966 hasta 2004) EMBASE (1988 hasta 2004) Psyclnfo (1985 hasta 2004) CINAHL (1982 hasta 2004) Pascal y Toxibase. También realizaron contactos para obtener estudios no publicados y en curso.

Incluyeron todos los ensayos clínicos aleatorizados (ECA) sobre tratamiento de la dependencia a benzodiazepinas (como única droga) en pacientes ambulatorios, independientemente de su tipo, de la dosis diaria y total y de la duración del tratamiento.

Los revisores evaluaron en forma independiente cada uno de los ensayos para su inclusión, calificando su calidad metodológica; y terminaron incluyendo ocho ensayos con un total de 458 participantes: cinco estudios realizados en Inglaterra, uno en Francia y dos en Estados Unidos.

* Alumna de la Carrera de Medicina del Instituto Universitario Hospital Italiano de Buenos Aires. 
Todos los participantes habían recibido una dosis estable de benzodiazepinas antes de iniciar los programas de interrupción de estas drogas. La duración de los estudios fue de cuatro a diecisiete semanas y algunos de ellos agregaron un seguimiento de varias semanas después de concluido el proceso de reducción de las benzodiacepinas. Fueron incluidos sujetos de 39 a 54 años de edad que hubieran consumido estos fármacos durante tres meses a un año; y hubieran intentado varias veces reducir o detener el consumo sin éxito.

\section{Tipo de comparaciones}

Un estudio comparó la reducción gradual versus la abstinencia abrupta; mientras que los otros siete compararon la reducción gradual ayudada por un tratamiento farmacológico adyuvante versus la reducción gradual junto a placebo.

Como fármaco adyuvante fueron utilizados: propranolol (dos estudios) antidepresivos tricíclicos, agonistas serotoninérgicos $5 \mathrm{HT} 1 \mathrm{a}$, hidroxicina, anticonvulsivantes y progesterona.

Debido a la heterogeneidad de los ámbitos, del diseño de los estudios, de la fuente y del formato de las intervenciones, no fue posible agrupar los datos para un metanálisis; lo que condujo a que fueran analizados en forma de una revisión narrativa.

Resultados

Todos los resultados de los estudios incluidos estuvieron de acuerdo en que la reducción gradual era preferible a la interrupción abrupta. Por ejemplo, la abstinencia progresiva (durante más de diez semanas) parece preferible a la abrupta, ya que el número de abandonos fue menor y los participantes consideraron más favorable el procedimiento.

\section{Cambio a benzodiacepinas de vida media larga}

Los resultados de esta revisión no avalan el cambio de una benzodiazepina de vida media corta a una de vida media larga antes de iniciar el proceso de reducción gradual -conducta muy recomendada por muchos expertos -, habiendo abordado esta cuestión sólo un ensayo²: de los 68 participantes inicialmente incluidos, sólo 45 completaron el estudio. Lo abandonaron diez de los asignados a lorazepam (de vida media corta) siete de los asignados a bromazepam y seis de los asignados a diazepam (ambos de vida media intermedia o larga). Cabe aclarar que dado que el ensayo contó con pocos participantes, su poder estadístico fue bajo, lo que puede explicar la ausencia de significación estadística.

\section{Uso coadyuvante de carbamacepina}

Los resultados de esta revisión sistemática informan que la carbamazepina tiene un valor potencial como intervención efectiva para la reducción gradual de una benzodiazepina hasta la interrupción de su uso. Si bien la carbamazepina ha demostrado beneficios moderados para disminuir la gravedad de los síntomas de abstinencia, se asoció a una mejoría significativa en la tasa de abstinencia, en particular en los pacientes que venían recibiendo dosis diarias mayores a $20 \mathrm{mg}$ de diazepam (o de sus equivalentes). Por ejemplo, en el estudio realizado por Schweizer y col. ${ }^{1}$ el $95 \%$ de los participantes tratados con carbamazepina se mantuvo libre de benzodiazepinas a las cinco semanas después de la reducción en comparación con el $62 \%$ de los participantes tratados con placebo $(p<0,03)$.

\section{Propranolol, dotiepina, buspirona y progesterona}

La función del propranolol en la abstinencia a las benzodiazepinas fue incierta; mientras que el agregado de un antidepresivo tricíclico (dotiepina) disminuyó la intensidad de los síntomas de abstinencia pero no aumentó la tasa de abstinencia de benzodiazepina al final del ensayo. La buspirona y la progesterona no lograron suprimir los síntomas de abstinencia de la benzodiazepina.

\section{Comentario}

Dados los resultados de la revisión sistemática que hemos resumido, es necesario realizar estudios controlados de mayor poder con el objetivo de investigar si el cambio de una benzodiazepina de vida media corta a la de una de vida media larga antes de iniciar el proceso de reducción gradual tiene repercusión sobre la intensidad de los síntomas de abstinencia. Otra interesante área de investigación incluye la de los posibles beneficios de la carbamazepina, para evaluar sus efectos adversos y determinar y consensuar su indicación clínica.

Por otro lado, sería de utilidad analizar otros enfoques de tratamiento que han sido sugeridos para el tratamiento de interrupción de las benzodiacepinas:

1) El flumazenil, un antagonista de los receptores de benzodiazepina que puede regular en forma retrógrada los receptores de la benzodiacepina; pero que, sin embargo, no cuenta con evidencia proveniente de ensayos controlados que avale su uso en esta situación clínica.

2) Los antidepresivos, que a partir de su capacidad para inhibir los receptores monoaminérgicos y para reducir los niveles de depresión y ansiedad, también pueden mostrar beneficios. Sin embargo, no hemos encontrado evidencia que avale su uso en esta situación.

3) Estrategias psicológicas para el tratamiento de la dependencia a estas drogas. Si bien se han descritos técnicas que podrían aplicarse con tal fin, como la relajación muscular progresiva y la meditación, los resultados obtenidos han sido poco esperanzadores.

Consideramos que es muy importante que los médicos que trabajamos en atención primaria tengamos un control estricto de la prescripción de las benzodiacepinas y que cuando las prescribamos, lo hagamos en el contexto de planes terapéuticos que incluyan objetivos a corto plazo, evaluando periódicamente su eficacia y fármaco-toxicidad. Por otro lado, ante la situación de encontrarnos frente a un paciente consumidor crónico y dependiente de estas drogas, es importante plantearnos (primero nosotros) y plantearle (luego a él) la necesidad de discontinuarlas. De acuerdo a los resultados de esta revisión, la mejor manera de lograrlo sería reduciendo gradualmente la dosis de las benzodiacepinas; con el uso eventual de carbamacepina como coadyuvante.

Volviendo a la paciente descripta en la viñeta clínica que inspiró este trabajo, aprovechamos para recordar al lector que los pacientes con insomnio ${ }^{3}$, especialmente el que aparece en el contexto de una situación de duelo, merecen un abordaje integral que escapa a los objetivos de este artículo pero que puede ser ampliado en otras lecturas que ya hemos publicado ${ }^{3,4}$.

Recibido el 10/04/09 y aceptado el 05/05/09.

Referencias

1. Schweizer E y col. Carbamazepin treatment in patients discontinuing long-term benzodiazepine therapy. effects on withdrawal severity and outcome. Archives of General Psychiatry 1991;48(5):448-52.

2. Murphy $S$ y col. A double-blind comparison of the effects of gradual withdrawal of lorazepam, diazepam and bromazepam in benzodiazepine dependence. British Journal of Psychiatry 1991; 158:511-6.

3.Botargues M. Para "quitarnos el sueño": ¿usamos adecuadamente las benzodiacepinas? Evid. actual. pract. ambul. 2005; 8:89-91. Disponible en URL: http://www.foroaps.org/files/2005_8_89_91.pdf

4. Chajud S y col. Actualización: Duelo. Eviden.actual.pract.ambul 2004; 7:110-113. Disponible en URL: http://www.foroaps.org/files/el\%20duelo.pd 